\title{
Immunohistochemical Biomarkers of Survival in Patients With Adenocarcinoma of the Uterine Cervix Receiving Chemoradiotherapy
}

\author{
YING-CHUN LIN ${ }^{1}$, RUI-YUN CHEN ${ }^{2}$, JI-AN LIANG ${ }^{1,3}$, YAO-CHING HUNG $^{3,4}$, LIAN-SHUNG YEH ${ }^{3,4}$, \\ WEI-CHUN CHANG ${ }^{3,4}$, WU-CHOU LIN ${ }^{4,5}$, YIN-YI CHANG ${ }^{4,5}$ and SHANG-WEN CHEN ${ }^{1,3,6}$ \\ ${ }^{1}$ Department of Radiation Oncology, China Medical University Hospital, Taichung, Taiwan, R.O.C.; \\ ${ }^{2}$ Department of Pathology, China Medical University Hospital, Taichung, Taiwan, R.O.C.; \\ ${ }^{3}$ Graduate Institute of Clinical Medical Science, School of Medicine, \\ College of Medicine, China Medical University, Taichung, Taiwan, R.O.C.; \\ ${ }^{4}$ Department of Obstetrics and Gynecology, China Medical University Hospital, Taichung, Taiwan, R.O.C.; \\ ${ }^{5}$ School of Chinese Medicine, College of Medicine, China Medical University, Taichung, Taiwan, R.O.C.; \\ ${ }^{6}$ Department of Radiology, School of Medicine, College of Medicine, Taipei Medical University, Taipei, Taiwan, R.O.C.
}

\begin{abstract}
Background/Aim: To determine the prognostic effects of immunohistochemical biomarkers for predicting chemoradiotherapy (CRT)-based treatment outcomes in patients with adenocarcinoma of the uterine cervix. Materials and Methods: This study included 42 patients receiving definitive CRT. According to the International Federation of Gynecology and Obstetrics staging system, 13, 21, and 8 patients were classified as having stage IB2, II, and III disease, respectively. Baseline immunohistochemical biomarkers, including those for hypoxia, cell proliferation, cell adhesion, immunogenicity, and evasion of apoptosis, were analyzed using tissue microarrays from biopsy specimens. Results: Myeloid cell leukemia-1 (MCL1) overexpression and the presence of pelvic lymph node metastasis were two prognostic factors for inferior cancer-specific survival. A higher H-score for c-MYC proto-oncogene, bHLH transcription factor (c-MYC) was associated with lower pelvic relapse-free survival. Conclusion: For patients with adenocarcinoma of the uterine cervix requiring definitive CRT, treatment outcomes can be stratified by the immunohistochemical biomarkers MCL1 and c-MYC for cancer death and local failure, respectively.
\end{abstract}

Adenocarcinoma (AC) of the uterine cervix constitutes approximately $10-20 \%$ of all uterine cervical carcinomas with a trend toward a rising incidence (1-3). Possible reasons

Correspondence to: Shang-Wen Chen, 2nd Yuh-Der Rd, North District, Taichung City, Taiwan, R.O.C. Tel: +886 4220521217450, e-mail: vincent1680616@yahoo.com.tw

Key Words: Cervical adenocarcinoma, chemoradiotherapy, immunohistochemistry, prognosis, myeloid cell leukemia-1. for this increase include obesity, nulliparity, and human papillomavirus-18 infection $(4,5)$. AC can be localized deep in the endocervical canal and easily be missed with the usual sampling in screening programs. Some studies indicated that $\mathrm{AC}$ and squamous cell carcinoma (SCC) behave differently in epidemiology (2-7), and have differential genomic expression $(8,9)$. In addition, they have diverse prognostic factors and patterns of failure after similar treatments $(4,6$, 10-13). Currently, most treatment knowledge of cervical AC comes from studies where the majority of patients had SCC. Therefore, molecular profiling of novel treatment strategies specifically for AC is imperative.

Given that chemoradiotherapy (CRT) has been the standard of care for patients with locally advanced cervical cancer worldwide, radioresistance or treatment failure is a clinically relevant problem. Patients with cervical AC primarily treated with radiotherapy have inferior outcomes compared with those with SCC (4, 12-14). As advances in molecular profiling have allowed for the identification of biomarkers of many biological characteristics in tumor cells, biomarkers in standard treatment are of interest for their potential role in the design of personalized therapeutic strategies targeting individual tumors. In cervical cancer, several biomarkers for RT-based treatment have been validated by patient survival or recurrence data $(15,16)$. These biomarkers fall into categories according to biological function including hypoxia, cell proliferation, cell adhesion, immunogenicity, and evasion of apoptosis (15). There is a great need to identify biomarkers since there are few studies on CRT-based prognostic factors specifically for cervical AC. Hence, this study was conducted to investigate the impact of pretreatment immunohistochemical (IHC) markers and clinical parameters on CRTbased treatment in these patients. 


\section{Materials and Methods}

Study population. This retrospective study included 42 patients newly diagnosed with AC of the uterine cervix between July 2009 and December 2015. All patients had undergone ${ }^{18} \mathrm{~F}$-fluorodeoxyglucose positron-emission tomography/computed tomography (PET/CT) for staging and had received allocated external-beam radiotherapy and intracavitary brachytherapy. Concurrent chemotherapy consisted of weekly administration of $40 \mathrm{mg} / \mathrm{m}^{2}$ of cisplatin. The eligibility criteria included patients with stage IB2 to IIIB disease in accordance with staging system of the International Federation of Gynecology and Obstetrics (FIGO) (17). Accordingly, 13, 21, and eight patients were classified as having stage IB2, II, and III disease, respectively. The median age of our patients was 55 years. Because PET/CT has high sensitivity and specificity in detecting the nodal status in cervical cancer, the diagnosis of pelvic lymph node (PLN) metastasis was based on PET/CT. Patients with para-aortic lymph node metastasis were excluded. In addition, we excluded patients who were histologically diagnosed with adenosquamous carcinoma. This study was approved by the local Institutional Review Board (CMUH107REC3-008). Patient characteristics are listed in Table I.

Immunohistochemistry. IHC biomarkers, namely endogenous hypoxic [glucose transporter 1 (GLUT1), carbonic anhydrase IX (CAIX), and hypoxia-inducible factor 1-alpha (HIF1 $\alpha)$, angiogenesis or metastasis ([vascular endothelial growth factor (VEGF), c-MET]), cell proliferation [epidermal growth factor receptor (EGFR), c-MYC, insulin-like growth factor 1 receptor (IGF1R)], cell to cell adhesion (E-cadherin, vimentin), evasion to apoptosis [B-cell lymphoma 2 (BCL2), BCL2-associated protein X (BAX), myeloid cell leukemia 1 (MCL1)], and immunogenic or inflammatory biomarkers [programmed cell death protein ligand 1 (PD-L1), tumor necrosis factor- $\alpha(\mathrm{TNF} \alpha)$, calretinin, galectin-9, and chemokine ligand 5 (CCL5)] were analyzed using tissue microarrays from incisional biopsy specimens before treatment. Each tumor was represented by one tissue core on a tissue microarray. Furthermore, 4- $\mu \mathrm{m}$-thick paraffin sections were deparaffinized and microwaved according to standard procedures before being processed for IHC staining. The antibodies for staining of biomarkers are detailed as follows: polyclonal rabbit antihuman Glut1 (1:200; GTX15309; GeneTex; Santa Barbara, CA, USA), liquid mouse monoclonal CAIX (1:200; clone TH22; Novocastra; Santa Barbara, CA, USA), monoclonal mouse antihuman VEGF (1:800; NB100-664; Novus Biologicals; Santa Barbara, CA, USA), HIF-1 $\alpha$ rabbit polyclonal (1:200; ab10625; Abcam; Santa Barbara, CA, USA), rabbit monoclonal EGFR (1:100; clone EP22; Zeta; Santa Barbara, CA, USA), liquid mouse monoclonal Bcl-2 (1:100; clone bcl-2/100/D5; Novocastra), monoclonal rabbit anti-Met (cMet) antibodies (1:200; EP1454Y; Abcam), monoclonal mouse IgG human c-MYC antibody (1:100; clone \#9E10; bio-techne, Devens, MA, USA), and monoclonal mouse IgG human MCL1 antibody (1:50; clone \#4B7; GeneTex), monoclonal rabbit IgG anti-human Bax antibody (clone \#E63; Santa Barbara, CA, USA), polyclonal goat IgG anti-human/mouse IGF-1R antibody (catalog \#391-GR; bio-techne), monoclonal rabbit $\mathrm{IgG}$ anti-human Vimentin antibody (clone \#SP20; Fremont, CA, USA), monoclonal rabbit IgG antihuman E-cadherin antibody (clone \#EP700Y; Fremont, CA, USA), polyclonal rabbit IgG anti-human/mouse TNF- $\alpha$ antibody (catalog \#GTX110520; GeneTex), polyclonal rabbit IgG anti-human/mouse calretinin antibody (catalog \#GTX111627; GeneTex), polyclonal
Table I. Patient characteristics $(n=42)$.

\begin{tabular}{|c|c|}
\hline Variable & Value \\
\hline \multicolumn{2}{|l|}{ Age, years } \\
\hline Median (range) & $55(33-77)$ \\
\hline \multicolumn{2}{|l|}{ FIGO stage } \\
\hline IB2 & $13(31 \%)$ \\
\hline IIA-IIB & $21(50 \%)$ \\
\hline IIIA-IIIB & $8(19 \%)$ \\
\hline \multicolumn{2}{|c|}{ Maximum tumor dimension, $\mathrm{cm}$} \\
\hline Mean \pm SD (range) & $5.7 \pm 1.1(3.9-8.6)$ \\
\hline \multicolumn{2}{|c|}{ Pelvic lymph node metastasis } \\
\hline Negative & $24(57 \%)$ \\
\hline Positive & $18(43 \%)$ \\
\hline \multicolumn{2}{|c|}{ Pretreatment hemoglobulin, g/dI } \\
\hline Mean \pm SD (range) & $10.3 \pm 3.0(3.5-14.3)$ \\
\hline \multicolumn{2}{|c|}{ Carcinoembryonic antigen, $\mathrm{ng} / \mathrm{dI}$} \\
\hline Mean \pm SD (range) & $36.2 \pm 22.9(0.4-528.8)$ \\
\hline \multicolumn{2}{|c|}{ External beam radiotherapy, Gy } \\
\hline \multicolumn{2}{|c|}{ Whole pelvis, Gy } \\
\hline Median (range) & $45(39.6-54)$ \\
\hline \multicolumn{2}{|c|}{ Bilateral parametrial boost, central shielding, Gy } \\
\hline Median (range) & $54(50.4-57.6)$ \\
\hline \multicolumn{2}{|c|}{ Pelvic lymph node boost, Gy } \\
\hline Median (range) & $64(60-66)$ \\
\hline \multicolumn{2}{|l|}{ Brachytherapy } \\
\hline \multicolumn{2}{|l|}{ 2-Dimensional* } \\
\hline Number of patients & 14 \\
\hline \multicolumn{2}{|c|}{ Cumulative EQD2 of point $\mathrm{A}, \mathrm{Gy}_{10}$} \\
\hline Mean \pm SD & $84.3 \pm 7.3$ \\
\hline \multicolumn{2}{|c|}{ 3-Dimensional brachytherapy\# } \\
\hline Number of patients & 28 \\
\hline \multicolumn{2}{|c|}{ Cumulative EQD2 of D90, $\mathrm{Gy}_{10}$} \\
\hline Mean \pm SD & $88.1 \pm 10.3$ \\
\hline
\end{tabular}

FIGO: International Federation of Gynecology and Obstetrics; EQD2: equivalent dose in $2 \mathrm{~Gy}$; HR-CTV: high-risk clinical target volume. *6 Gy to point A per session for 5 courses. "HR-CTV $>6.5$ Gy per session for 5 courses.

rabbit IgG anti-human galectin-9 antibody (catalog \#GTX127352; GeneTex), polyclonal rabbit IgG anti-human/mouse CCL5 antibody (NBP1-19769; bio-techne).

The staining slides were scored by two pathologists blinded to the clinical outcome. Except for PD-L1, IHC results of the aforementioned biomarkers were scored by a semiquantitative approach used to assign an $\mathrm{H}$-score to tumor samples (18). The $\mathrm{H}-$ score takes into consideration the staining intensity in conjunction with the percentage of cells staining positively. Staining intensity was graded as $0,1,2$, and 3 corresponding to negative, mild, moderate, and strong staining, respectively. The percentage of positively stained tumor cells was estimated by the observers. The total number of neoplastic cells in the field and the number of neoplastic cells stained at each intensity were counted. The following formula was applied: $\mathrm{H}$-score $=[\%$ of cells stained at intensity category 1 (neoplastic cells with mild staining) $\times 1]+[\%$ of cells stained at intensity category 2 (neoplastic cells with moderate staining) $\times 2]+[\%$ of cells stained at intensity category 3 (neoplastic cells with strong staining) $\times 3$ ]. Accordingly, the $\mathrm{H}$-scores were calculated, ranging from 0 to 300 , with 300 being equal to $100 \%$ of tumor cells stained strongly (3+). 
Tumor biomarker PD-L1 was evaluated through IHC staining using the DAKO clone 22C3 pharmDx kit (DAKO, Carpinteria, CA, USA). PD-L1 expression was scored according to the combined positive score, which is the number of PD-L1 -staining cells (tumor cells, lymphocytes, macrophages) at any intensity divided by the total number of viable tumor cells, multiplied by 100 (19).

Treatment. The treatment was described previously $(20,21)$. All patients were treated with intensity-modulated RT. The total dose applied to the pelvis was $45 \mathrm{~Gy}$, administered in 25 fractions over a 5-week period. Following pelvic irradiation, the bilateral parametrium was boosted from 50.4 to $54 \mathrm{~Gy}$.

After adequate tumor regression, high-dose-rate intracavitary brachytherapy was performed once or twice a week using an ${ }^{192} \mathrm{Ir}$ remote afterloading technique concurrently with pelvic irradiation or parametrial boosting. Before January 2013, the standard prescribed dose for each session of brachytherapy was $6.0 \mathrm{~Gy}$ to Point A, with five sessions. After January 2013, 28 patients were treated with three-dimensional image-based brachytherapy according to the recommendations of the Groupe Européen de Curietherapie and the guidelines specified by the European Society for Radiotherapy and Oncology (22). The details of the cumulative dose are summarized in Table I.

Chemotherapy consisted of weekly $40 \mathrm{mg} / \mathrm{m}^{2}$ doses of cisplatin, administered intravenously to a total dose of $60 \mathrm{mg}$.

Follow-up. After completion of RT, patients were regularly followed-up every 2 months for the first year, and every 3 to 4 months thereafter. Besides a routine pelvic examination, the serum level of the tumor marker carcinoembryonic antigen was examined during each follow-up. Additionally, a radiographic examination was performed every 6 months. Patients exhibiting symptoms of central-pelvic recurrence underwent a salvage hysterectomy or pelvic exenteration, if feasible. Patients with distant metastasis were treated with systemic chemotherapy.

Statistical analysis. To examine correlations between the aforementioned parameters and tumor recurrence, receiver operating characteristic (ROC) curves were constructed to evaluate the optimal predictive performance among the various IHC and clinical parameters, such as maximum tumor dimension and pretreatment hemoglobulin $(\mathrm{Hb})(23)$. In addition, binary logistic regression analysis was performed to determine the independent factors among all IHC biomarkers for predicting clinical outcomes. The quantitative differences between $\mathrm{H}$-scores of the biomarkers and clinical parameters were examined using the Mann-Whitney $U$-test. The outcome endpoints were cancerspecific survival (CSS), distant metastasis-free survival (DMFS), and pelvic relapse-free survival (PRFS), all of which were calculated using the Kaplan-Meier method. The log-rank test and Cox regression analysis were performed to examine the effects of explanatory variables on these endpoints. The stage, age, PLN status, maximum tumor dimension, $\mathrm{Hb}$ level, and predictive IHC markers were included for multivariate analysis. Patient survival was measured from the date of initiation of RT to the last followup. Two-tailed tests were used, and values $p<0.05$ was considered statistically significant. All calculations were performed using SPSS, Version 13.0 for Windows (SPSS Inc., Chicago, IL, USA).

\section{Results}

Treatment outcomes. After a median follow-up duration of 48 months (range=7-120), 29 patients were alive and 13 patients had died of cancer progression. Twenty-three patients had no evidence of disease progression. Eight out of the 19 patients with disease progression had infield recurrence, five had distant metastasis, and six had both. None of the 14 patients with infield recurrence experienced sole relapse of PLN. In summary, 14 patients had local residual or recurrent tumors at primary sites, whereas 11 patients experienced distant metastasis. Seven patients underwent salvage operation for residual or recurrent primary tumor, whereas 10 received systemic chemotherapy after the diagnosis of distant metastasis.

Predictive ability of IHC biomarkers. All aforementioned IHC biomarkers were retrieved. Table II lists the biomarkers and the area under the ROC curve. The c-MYC H-score most accurately predicted the presence of local residual or recurrent tumors $(\mathrm{AUC}=0.71, p=0.03$ ). Logistic regression analysis showed the c-MYC H-score had the highest predictive value in the cohort [odds ratio $(\mathrm{OR})=1.30,95 \%$ confidence interval $(\mathrm{CI})=1.001-1.061 ; p=0.045]$. Based on Youden's index, we found that an optimal cut-off for the cMYC H-score was 27 (AUC $=0.68, p=0.06$ ).

The ROC curves showed that the MCL1 H-score was the sole biomarker that predicted death from cancer $(\mathrm{AUC}=0.69$, $p=0.06$ ). In logistic regression analysis, however, the MCL1 $\mathrm{H}$-score failed to attain statistical significance probably due to restricted events $(p=0.058)$. When using an optimal cutoff of 115 for the MCL1 H-score, the AUC for death from cancer was $0.69(p=0.055)$.

None of the other IHC biomarkers, including those for hypoxia, cell adhesion, or immunogenicity biomarkers, appeared to be prognostic for the three study endpoints for this cohort. Therefore, the MCL1 and c-MYC H-scores, combined with age, stage, maximum tumor dimension, pretreatment $\mathrm{Hb}, \mathrm{PLN}$ status, and brachytherapy schemes (2D versus $3 \mathrm{D}$ ) were selected for multivariate Cox regression model for survival analyses.

Prognostic factors for CSS, PRFS, and DMFS. As summarized in Table III, Cox regression analysis indicated that the MCL1 H-score (hazard ratio $(\mathrm{HR})=12.82,95 \%$ $\mathrm{CI}=1.53-107.38 ; p=0.019)$ and presence of PLN metastasis $(\mathrm{HR}=4.49, \mathrm{CI}=1.21-16.68 ; p=0.025)$ were two factors prognostic for CSS. As depicted in Figure 1, the 4-year CSS of patients with and without PLN disease was $52 \%$ and $85 \%$ $(p=0.02)$, and among patients who had tumors with a MCL1 $\mathrm{H}$-score $>115$ and $\leq 115$, it was $40 \%$ and $84 \%$, respectively $(p=0.004)$. The trend remained statistically significant when dichotomizing the patients with a cutoff using the median 

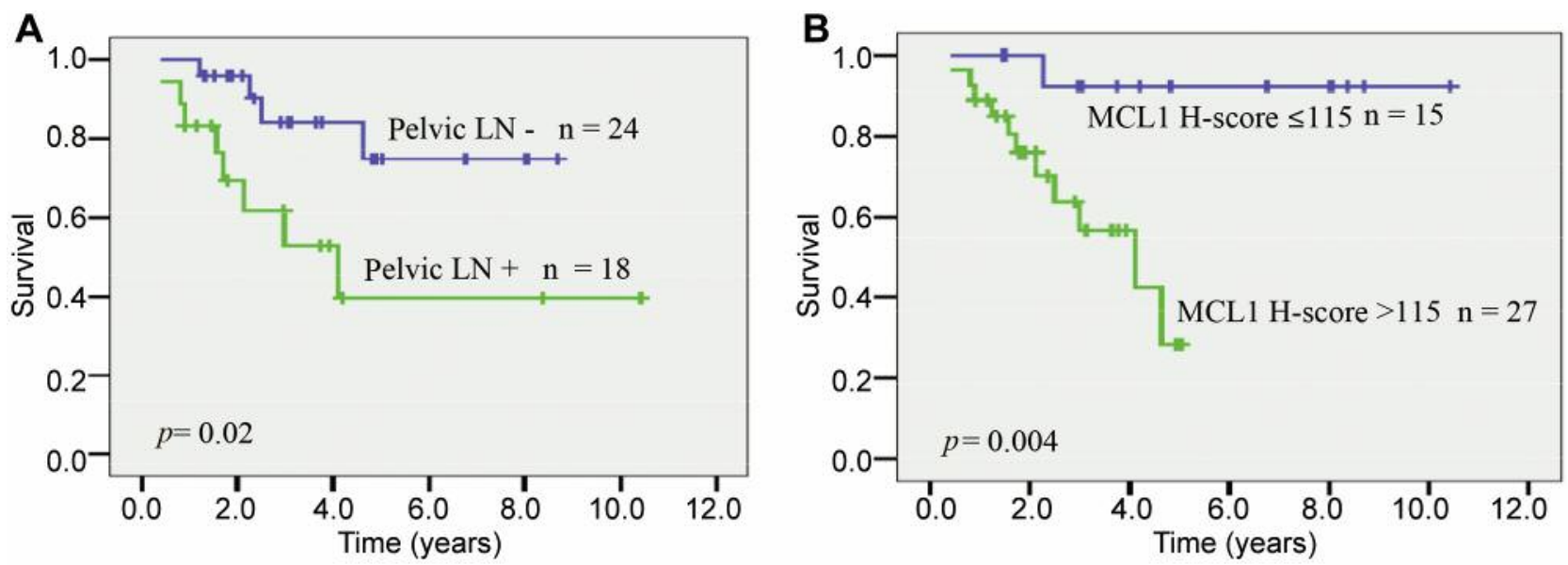

Figure 1. Cancer-specific survival in patients who had tumors with and without pelvic lymph node (LN) metastasis (A), and according to myeloid cell leukemia-1 (MCL1) H-score (>115 vs. $\leq 115)(B)$.

MCL1 H-score of 130. The 4-year CSS of patients in high and low MCL1 expression groups was $60 \%$ and $79 \%$, respectively $(p=0.048)$.

Cox regression analysis disclosed that the c-MYC H-score was the sole predictor of poor PRFS $(\mathrm{HR}=1.03, \mathrm{CI}=1.01$ $1.05 ; p=0.011)$. Using the optimal cut-off of 27 for the cMYC score, the 4-year PRFS of patients with tumors with high and low c-MYC H-scores was 52\% and $77 \%$, respectively ( $p=0.048$, Figure 2$)$.

None of the IHC biomarkers were prognostic for DMFS; the major determinants of poor DMFS were PLN disease $(\mathrm{HR}=5.41, \mathrm{CI}=1.47-19.86 ; p=0.011)$ and FIGO stage III disease $(\mathrm{HR}=3.38, \mathrm{CI}=1.17-9.79 ; p=0.02)$. In multivariate analysis, age, tumor size, pretreatment $\mathrm{Hb}$, and brachytherapy schemes were not identified as independent prognostic factors for the aforementioned endpoints.

Quantitative differences between MCL1 or c-MYC H-scores and clinical parameters. Using the Mann-Whitney $U$-test, an association analysis was carried out to investigate the quantitative difference between MCL1 and c-MYC H-scores according to dichotomized clinical parameters including stage, PLN status, tumor dimension (median value of 5.6 $\mathrm{cm}$ ), and pretreatment $\mathrm{Hb}$ (median value of $10 \mathrm{gm} / \mathrm{dI}$ ). As shown in Figure 3A, patients presenting with pretreatment $\mathrm{Hb}<10 \mathrm{~g} / \mathrm{dl}$ had tumors with a significantly higher mean MCL1 H-score (26.6 vs. 17.7, $p=0.021)$. In addition, a lower $\mathrm{Hb}$ was associated with tumors having a higher mean vimentin to E-cadherin ratio ( $0.15 v s .0 .05, p=0.037$; Figure 3B). MCL1 and c-MYC H-scores were not related to stage, PLN status, or maximum tumor dimension. None of the clinical parameters or IHC biomarkers, including GLUT1 and HIF1 $\alpha$, were associated with c-MYC H-score.

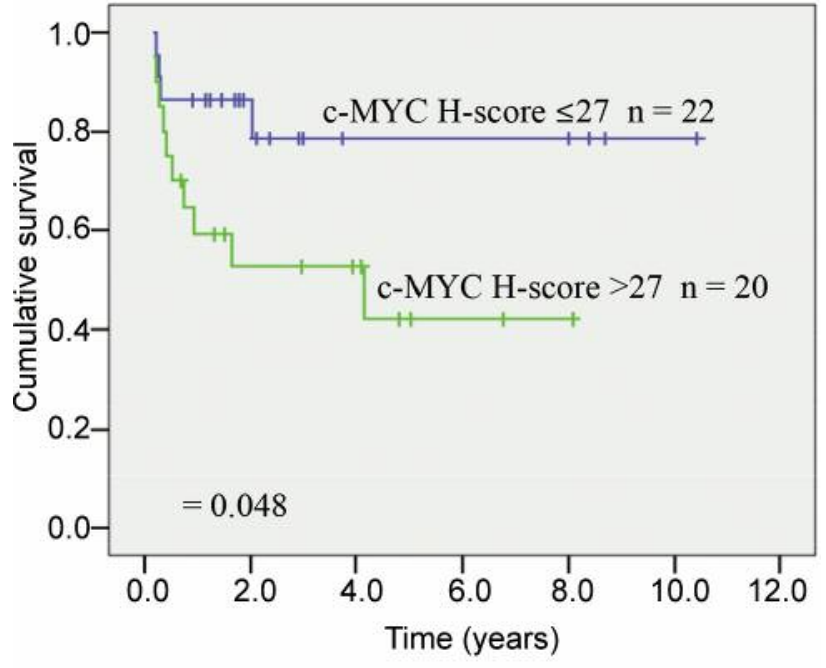

Figure 2. Pelvic relapse-free survival in patients according to tumor MYC proto-oncogene, bHLH transcription factor (c-MYC) H-score (>27 vs. $\leq 27)$.

Impact of combined overexpression of MCLI and C-MYC on survival. Because a recent study disclosed that coamplification of $c-M Y C$ and $M C L 1$ increases cancer stem cells in chemotherapy-resistant triple-negative breast cancer (24), the impact of combined MCL1 and c-MYC overexpression on survival was analyzed. Using the optimal values for both biomarkers, 18 tumors were identified as having cooverexpression. As shown in Figure 4, the 4-year CSS of patients with tumors with and without co-overexpression was $54 \%$ and $83 \%(p=0.026)$. The 4-year PRFS of patients with tumors with and without co-overexpression was 59\% and $73 \%$ $(p=0.15)$, whereas the 4-year DMFS for the two groups was 


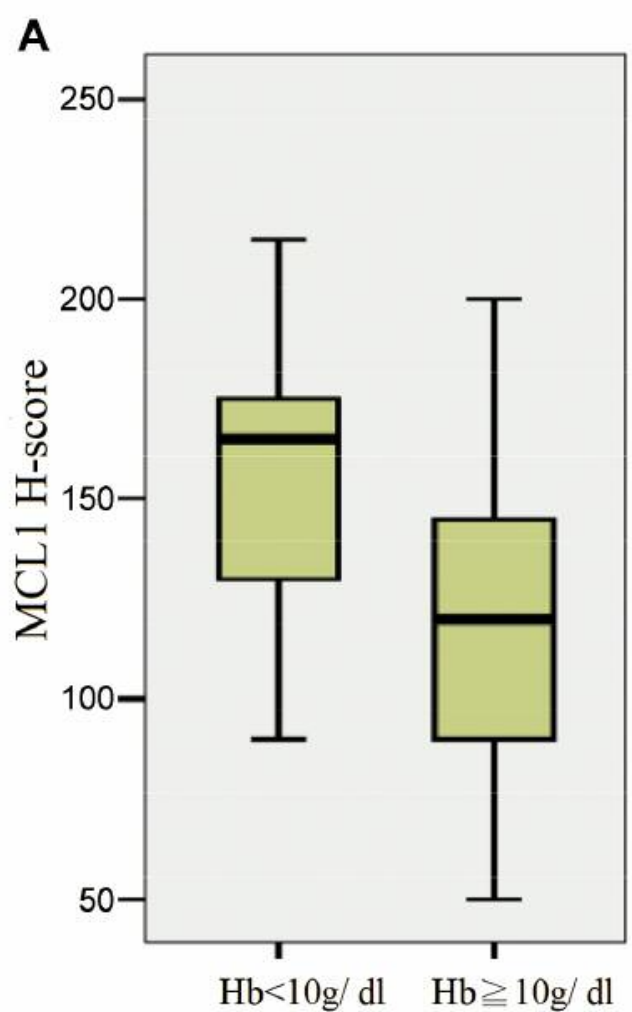

B

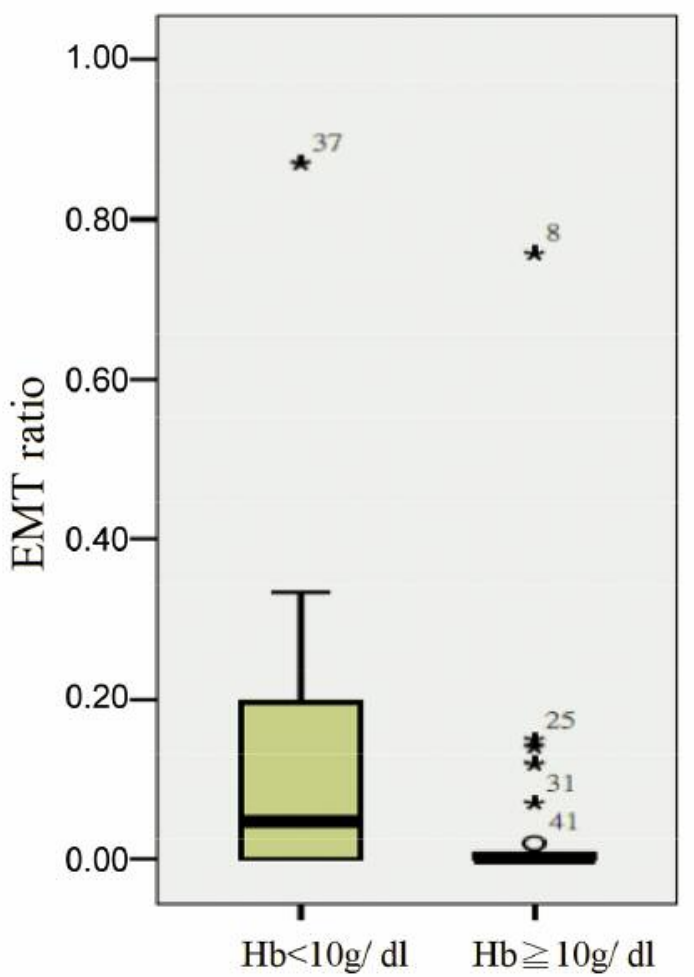

Figure 3. Myeloid cell leukemia-1 (MCL1) H-score (A), and vimentinto-E-cadherin (EMT) ratio according to pretreatment hemoglobulin $(B)$ $(p=0.021$ and $p=0.037$, respectively).
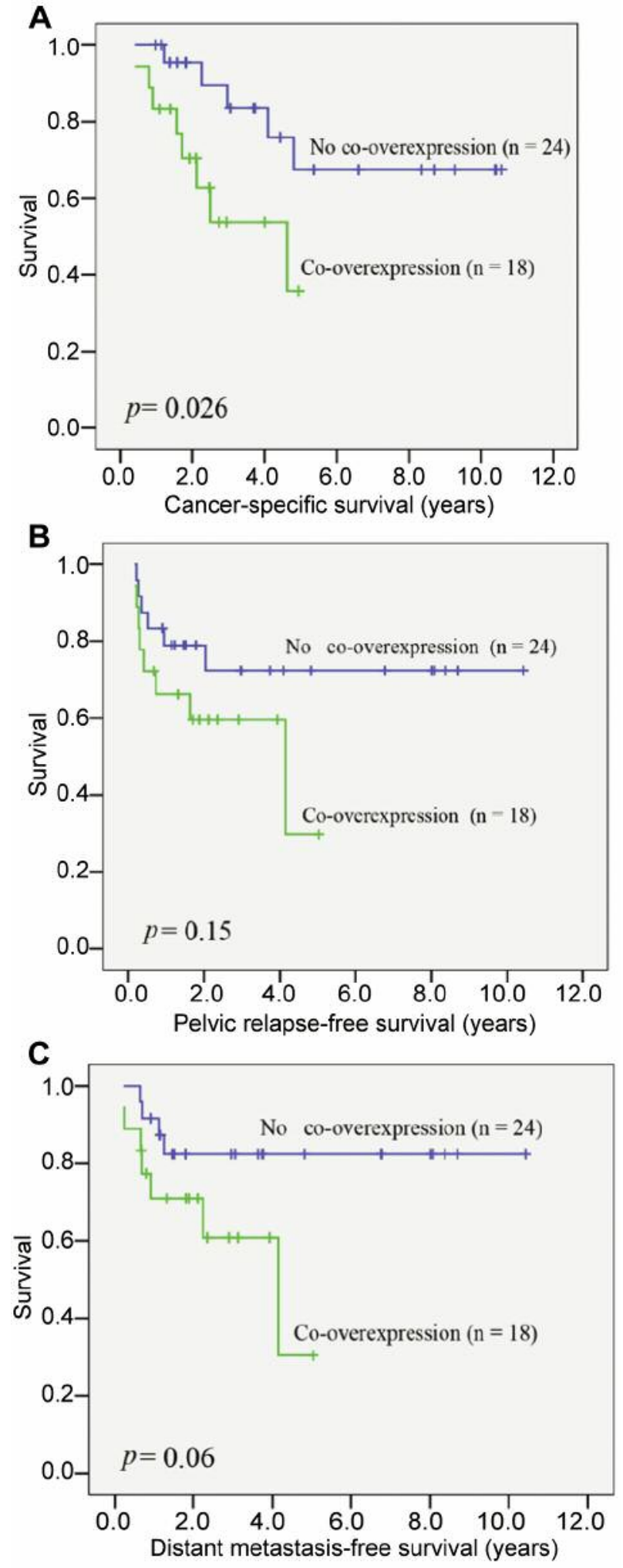

Figure 4. Cancer-specific (A), pelvic relapse-free (B) and distant metastasis-free $(C)$ survival curves of patients with tumors with and without co-overexpression of myeloid cell leukemia-1 (MCL1) and MYC proto-oncogene, bHLH transcription factor (c-MYC). 
Table II. Predictive immunohistochemical and clinical parameters and the area under the receiver operating characteristic curve (AUC).

\begin{tabular}{|c|c|c|c|c|c|c|}
\hline \multirow[b]{2}{*}{ Variable } & \multicolumn{2}{|c|}{ Cancer death } & \multicolumn{2}{|c|}{ Local failure } & \multicolumn{2}{|c|}{ Distant metastasis } \\
\hline & AUC & $p$-Value & AUC & $p$-Value & AUC & $p$-Value \\
\hline MCL1 H-score & $0.69 \pm 0.09$ & 0.06 & $0.61 \pm 0.09$ & 0.25 & $0.60 \pm 0.10$ & 0.32 \\
\hline c- MYC H-score & $0.59 \pm 0.10$ & 0.33 & $0.71 \pm 0.08$ & 0.03 & $0.49 \pm 0.11$ & 0.95 \\
\hline EGFR H-score & $0.38 \pm 0.10$ & 0.19 & $0.53 \pm 0.07$ & 0.77 & $0.34 \pm 0.10$ & 0.74 \\
\hline PD-L1 combined positive score & $0.44 \pm 0.10$ & 0.51 & $0.64 \pm 0.09$ & 0.15 & $0.44 \pm 0.11$ & 0.54 \\
\hline TNF $\alpha$ H-score & $0.42 \pm 0.10$ & 0.43 & $0.38 \pm 0.09$ & 0.22 & $0.41 \pm 0.09$ & 0.39 \\
\hline Calretinin H-score & $0.43 \pm 0.10$ & 0.48 & $0.52 \pm 0.09$ & 0.81 & $0.39 \pm 0.10$ & 0.29 \\
\hline Maximum tumor dimension & $0.60 \pm 0.10$ & 0.30 & $0.50 \pm 0.10$ & 0.97 & $0.62 \pm 0.10$ & 0.26 \\
\hline Pretreatment hemoglobulin & $0.49 \pm 0.10$ & 0.88 & $0.41 \pm 0.10$ & 0.34 & $0.45 \pm 0.11$ & 0.62 \\
\hline Pretreatment serum CEA & $0.56 \pm 0.10$ & 0.55 & $0.63 \pm 0.09$ & 0.18 & $0.59 \pm 0.09$ & 0.38 \\
\hline
\end{tabular}

CEA: Carcinoembryonic antigen; EGFR: epidermal growth factor receptor; MCL1: myeloid cell leukemia-1; c-MYC: MYC proto-oncogene, bHLH transcription factor; PD-L1: programmed cell death protein ligand 1; TNF $\alpha$ : tumor necrosis factor- $\alpha$.

Table III. Multivariate analyses using Cox regression analysis for cancer-specific (CSS), pelvic relapse-free (PRFS), and distant metastasis-free (DMFS) survival.

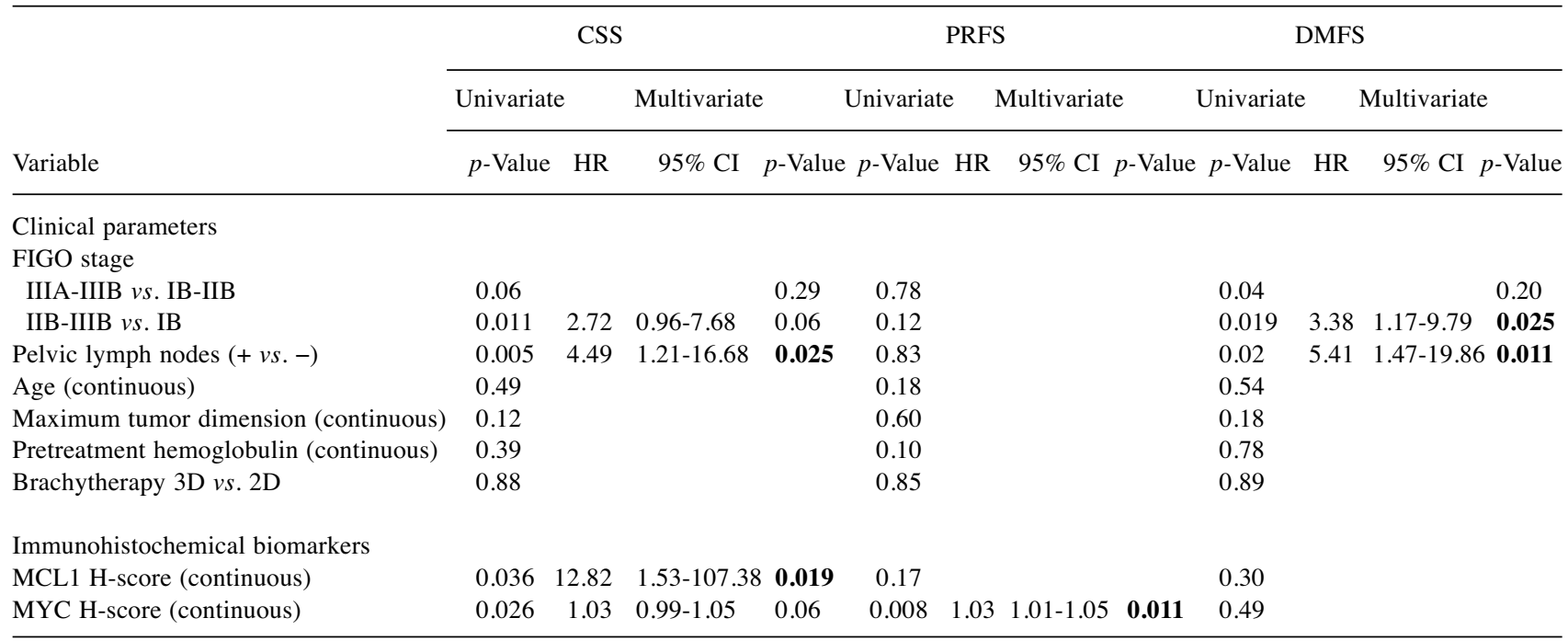

HR: Hazard ratio; CI: confidence interval; FIGO: International Federation of Gynecology and Obstetrics. Cox regression model with stepwise procedure was adopted to identify the prognostic factors. Statistically significant results in multivariate analysis are shown in bold.

$60 \%$ and $83 \%(p=0.06)$ (Figure 4$)$. However, there was no statistically significant trend of quantitative differences between co-overexpression and the other IHC biomarkers, including hypoxia or biomarkers of epithelial-mesenchymal transition (EMT). In addition, no association was found between co-overexpression and clinical parameters. 1

\section{Discussion}

An understanding of cancer phenotypes from genomic expression, IHC profiling, or imaging studies allows oncologists to use individualized therapy. Given that there are significant differential genomic expression changes between AC and SCC of the uterine cervix, the two tumor types behave differently $(4,8,9)$, and some studies have indicated that patients with cervical AC experience inferior CRT-based treatment outcomes $(4,12-14)$, there is a need to explore the biological mechanisms or tumor microenvironment specifically for patients with AC. To date, no comprehensive IHC studies for this specific tumor type are available for clinical practice. Our work here was a pilot study to compare wide-ranging quantitative IHC biomarkers in predicting the outcomes of patients with locally advanced cervical AC receiving definitive CRT. The assessment of 
various biomarkers revealed that MCL1 and c-MYC overexpression played roles in inferior CSS and PRFS, respectively. In addition, co-overexpression of the two markers was associated with lower CSS. Our findings revealed that certain biological characteristics of the tumors might supplement well-known clinical prognostic factors in predicting CRT-based treatment outcomes. Before initiating a novel therapeutic strategy for cervical AC, validation studies are required to confirm the findings.

In a recent review of the majority of studies on biomarkers for cervical SCC, the authors suggested that the most promising targets are apoptosis proteins, $\triangle \mathrm{Np} 73$ and BCL2; and hypoxia-related proteins galectin-1 and HIF $1 \alpha$, which are associated with radioresistance or poorer prognosis (15). Unlike the results of published results on cervical SCC, none of the studied IHC biomarkers representing hypoxia, cell adhesion, or immunogenicity appeared to be prognostic for the study endpoints. We identified MCL1, an anti-apoptotic member of the BCL2 family of apoptosis-regulating proteins, as an independent factor for cancer death. MCL1 overexpression has been reported in some hematological cancer and solid tumors (25). MCL1 blocks the progression of apoptosis by binding the pro-apoptotic proteins BCL2 homologous antagonist killer (BAK) and BAX, which are capable of forming pores in the mitochondrial membrane, allowing the release of cytochrome c into the cytoplasm $(26,27)$. Although our data showed the MCL1 H-score did not correlate with the pretreatment $\mathrm{Hb}$ by Spearman's correlation test (coefficient $=-0.25, p=0.12$ ), an $\mathrm{Hb}$ level of less than $10 \mathrm{~g} / \mathrm{dl}$ was associated with higher MCL1 expression. Alternatively using the median cut-off of MCL1, the pretreatment $\mathrm{Hb}$ value was also inversely correlated with MCL1 expression $(11.2 \mathrm{~g} / \mathrm{dI}$ in the low expression group $v s .9 .3 \mathrm{~g} / \mathrm{dl}$ in the high expression group, $p=0.04)$. Tumor hypoxia may directly contribute to the radioresistance of cancer. Some articles suggested the impact of pretreatment $\mathrm{Hb}$ or blood transfusion on CRT outcome in patients with cervical cancer (23, 28). In anemic patients, tumor oxygenation is compromised due to a reduced oxygen transport capacity of the blood. Accordingly, a direct association between hypoxia and anemia appears likely (28). Given none of the other IHC parameters, including the hypoxia markers, was significantly related to MCL1 expression, it would be interesting to know whether overexpression of MCL1 was the chicken or the egg for tumor hypoxia, or the engine of proliferation. Because MCL1 is not a biomarker for tumor hypoxia, the interplay between MCL1 and other survival pathways merits further studies.

The $c-M Y C$ oncogene is overexpressed in the majority of human cancers and contributes to the cause of at least $40 \%$ of tumors (29). $c-M Y C$ drives the metabolic changes which are important to support the increased need for nucleic acids, proteins and lipids necessary for rapid cellular proliferation (30). However, $c-M Y C$ expression is tightly regulated, and its level of expression is influenced at the transcriptional level by a number of transcriptional regulatory motifs (31). Several studies have shown that overexpression of $c-M Y C$ contributed to cancer radioresistance (32-36). c-MYC is involved in the repair of DNA double-strand breaks through the regulation of non-homologous end joining and homologous recombination repair (32). In addition, c-MYC promotes radioresistance through transcriptional activation of $C H E K 1$ and $C H E K 2$ checkpoint kinases through direct binding to the $C H K 1$ and $C H K 2$ promoters in a stem celllike population of nasopharyngeal cancer cells (33). Furthermore, a metabolism-associated or radioresistancerelated pathway, such as $\mathrm{HIF} 1 \alpha$, has been reported to have dramatic effects on c-MYC function (37). As our study revealed that HIF1 $\alpha$ and other IHC markers were not statistically associated with c-MYC H-scores, this implied that the mechanism for inferior tumor control by c-MYC overexpression is not directly linked to the Warburg effect (30). Therefore, c-MYC expression confers a molecular mechanism of radioresistance which might be complex and needs to be investigated further.

On the other hand, because MCL1 has a protective role in delaying apoptosis induced by $c-M Y C$ overexpression (38), and c-MYC and MCL1 enhance the stem cell-like potential of breast tumors via the hypoxia pathway (24), further clinical investigations are required to confirm the clinical impact of co-overexpression for other cancer types. To our knowledge, we are the first to show a cooperative role of cMYC and MCL1 in predicting CSS for patients with cervical AC. Furthermore, co-overexpression had a marginal impact on DMFS. Although quantitative differences between cooverexpresssion and hypoxia or stemness pathways were not found in our data as previously described (24), this novel discovery warrants additional molecular investigations.

EMT plays an important role in tumor invasion, metastasis, and prognosis, including cervical cancer $(9,39)$. Two hallmark EMT proteins, E-cadherin and vimentin, are tightly controlled during EMT through multiple signal transduction pathways. In this study, we applied the continuous values of the H-score rather than grading of the immunoreactive score to quantify the staining intensity of E-cadherin or vimentin. Therefore, the role of EMT has been examined when stratifying the CRT outcome. Despite the results showing that the three EMT parameters were not associated with the outcome, the finding that a lower $\mathrm{Hb}$ level was associated with tumors having a higher mean vimentin to E-cadherin ratio might provide clinical insight. Some in vivo and in vitro studies of tumor cells of different origins indicated that hypoxia marker HIF1 $\alpha$ may be directly involved in the down-regulation of E-cadherin through up-regulation of the EMT-inducing transcription factors SNAIL and TWIST $(40,41)$. 
The findings of this study should be interpreted cautiously because they represent a retrospective study at a single institution. External validation studies using an independent data set and a large sample size are necessary to confirm these findings. Furthermore, the precise molecular pathway that MCL1 or c-MYC overexpression confers to poor CRT-based outcomes could not be clarified through association studies between IHC biomarkers and clinical parameters. Additional molecular studies are recommended to elucidate the underlying biological mechanisms, as well as the interplay with other survival pathways. Finally, the association between DNA sequencing or transcriptomes and the protein product should be investigated to outline a comprehensive biological mechanism of radioresistance or distant metastasis for these patients. For example, whether the coamplification of $M C L 1$ and $c$-MYC genes causes the overexpression of the two IHC markers should be verified. Nevertheless, the strengths of this study include the uniform treatment strategies, and wide-ranging analyses of the IHC biomarkers. Our findings provide an indication that future studies can clarify the mechanisms related to failure of CRT. In addition, this study took the initial step to enable the tailoring of CRT to specific biological characteristics of patients with cervical AC. Future studies should include information on next-generation sequencing and enroll patients prospectively. Oncologists might then be able to then assess the feasibility of personalized therapy for high-risk patients, such as salvage surgery, dose escalation schemes, and a novel combination therapy.

\section{Conclusion}

For patients with $\mathrm{AC}$ of the uterine cervix requiring definitive CRT, CSS can be stratified by the IHC biomarker MCL1, and the presence of PLN metastasis. The c-MYC $\mathrm{H}$-score most accurately predicted the presence of local residual or recurrent tumors after CRT. In addition, cooverexpression of MCL1 and c-MYC led to more cancer deaths. External validation studies are required to verify our findings.

\section{Conflicts of Interest}

All Authors declare no conflicts of interest in regard to this study.

\section{Authors' Contributions}

YC Lin, RY Chen, and SW Chen were responsible for the study design. All Authors collected the data. YC Lin, RY Chen, and SW Chen performed statistical analyses, interpreted data, and drafted the article. All Authors provided some intellectual content. SW Chen and YC Lin approved the version to be submitted. All Authors read and approved the final article.

\section{Ethical Approval}

This study was approved by a local Institutional Review Board [CMUH 107-REC3-008].

\section{Acknowledgements}

This study is supported in part by grants from Ministry of Science and Technology in Taiwan (MOST107-2314-B-038-028 and MOST105-2314-B-039-023) and China Medical University Hospital (DMR-107-186, DMR-108-201, and CMU104-S-03). The funders had no role in the study design, data collection and analysis, decision to publish, or preparation of the manuscript. No additional external funding was received for this study.

\section{References}

1 Mathew A and George PS: Trends in incidence and mortality rates of squamous cell carcinoma and adenocarcinoma of cervix-worldwide. Asian Pac J Cancer Prev 10(4): 645-650, 2009. PMID: 19827887.

2 Smith HO, Tiffany MF, Qualls CR and Key CR: The rising incidence of adenocarcinoma relative to squamous cell carcinoma of the uterine cervix in the united states - a 24-year population-based study. Gynecol Oncol 78(2): 97-105, 2000. PMID: 10926787. DOI: 10.1006/gyno.2000.5826

3 Sherman ME, Wang SS, Carreon J and Devesa SS: Mortality trends for cervical squamous and adenocarcinoma in the united states. Relation to incidence and survival. Cancer 103(6): 12581264, 2005. PMID: 15693030. DOI: 10.1002/cncr.20877

4 Gien LT, Beauchemin MC and Thomas G: Adenocarcinoma: A unique cervical cancer. Gynecol Oncol 116(1): 140-146, 2010. PMID: 19880165. DOI: 10.1016/j.ygyno.2009.09.040

5 Bosch FX, Manos MM, Muñoz N, Sherman M, Jansen AM, Peto J, Schiffman MH, Moreno V, Kurman $\mathrm{R}$ and Shan KV: Prevalence of human papillomavirus in cervical cancer: A worldwide perspective. JNCI: Journal of the National Cancer Institute 87(11): 796-802, 1995. PMID: 7791229.

6 Hopkins MP and Morley GW: A comparison of adenocarcinoma and squamous cell carcinoma of the cervix. Obstet Gynecol 77(6): 912-917, 1991. PMID: 11240740.

7 Castellsague X, Diaz M, de Sanjose S, Munoz N, Herrero R, Franceschi S, Peeling RW, Ashley R, Smith JS, Snijders PJ, Meijer CJ, Bosch FX and International Agency for Research on Cancer Multicenter Cervical Cancer Study Group: Worldwide human papillomavirus etiology of cervical adenocarcinoma and its cofactors: Implications for screening and prevention. J Natl Cancer Inst 98(5): 303-315, 2006. PMID: 16507827. DOI: 10.1093/jnci/djj067

8 Contag SA, Gostout BS, Clayton AC, Dixon MH, McGovern RM and Calhoun ES: Comparison of gene expression in squamous cell carcinoma and adenocarcinoma of the uterine cervix. Gynecol Oncol 95(3): 610-617, 2004. PMID: 15581972. DOI: $10.1016 /$ j.ygyno.2004 .08.021

9 Cancer Genome Atlas Research Network; Albert Einstein College of Medicine; Analytical Biological Services; Barretos Cancer Hospital; Baylor College of Medicine; Beckman Research Institute of City of Hope; Buck Institute for Research on Aging; Canada's Michael Smith Genome Sciences Centre; Harvard Medical School; 
Helen F. Graham Cancer Center \&Research Institute at Christiana Care Health Services; HudsonAlpha Institute for Biotechnology; ILSbio, LLC; Indiana University School of Medicine; Institute of Human Virology; Institute for Systems Biology; International Genomics Consortium; Leidos Biomedical; Massachusetts General Hospital; McDonnell Genome Institute at Washington University; Medical College of Wisconsin; Medical University of South Carolina; Memorial Sloan Kettering Cancer Center; Montefiore Medical Center; NantOmics; National Cancer Institute; National Hospital, Abuja, Nigeria; National Human Genome Research Institute; National Institute of Environmental Health Sciences; National Institute on Deafness \&Other Communication Disorders; Ontario Tumour Bank, London Health Sciences Centre; Ontario Tumour Bank, Ontario Institute for Cancer Research; Ontario Tumour Bank, The Ottawa Hospital; Oregon Health \&Science University; Samuel Oschin Comprehensive Cancer Institute, Cedars-Sinai Medical Center; SRA International; St Joseph's Candler Health System; Eli \&Edythe L. Broad Institute of Massachusetts Institute of Technology \&Harvard University; Research Institute at Nationwide Children's Hospital; Sidney Kimmel Comprehensive Cancer Center at Johns Hopkins University; University of Bergen; University of Texas MD Anderson Cancer Center; University of Abuja Teaching Hospital; University of Alabama at Birmingham; University of California, Irvine; University of California Santa Cruz; University of Kansas Medical Center; University of Lausanne; University of New Mexico Health Sciences Center; University of North Carolina at Chapel Hill; University of Oklahoma Health Sciences Center; University of Pittsburgh; University of São Paulo, Ribeir ão Preto Medical School; University of Southern California; University of Washington; University of Wisconsin School of Medicine \&Public Health; Van Andel Research Institute; Washington University in St Louis: Integrated genomic and molecular characterization of cervical cancer. Nature 543(7645): 378-384, 2017. PMID: 28112728. DOI: $10.1038 /$ nature21386

10 Eifel P, Burke T, Morris M and Smith T: Adenocarcinoma as an independent risk factor for disease recurrence in patients with stage ib cervical carcinoma. Gynecol Oncol 59(1): 38-44, 1995. PMID: 7557613. DOI: 10.1006/gyno.1995.1265

11 Lai C-H, Hsueh S, Hong J-H, Chang T-C, Tseng C-J, Chou H-H, Huang K-G and Lin J-D: Are adenocarcinomas and adenosquamous carcinomas different from squamous carcinomas in stage ib and ii cervical cancer patients undergoing primary radical surgery? Int J Gynecol Cancer 9(1): 28-36, 1999. PMID: 11240740.

12 Huang YT, Wang CC, Tsai CS, Lai CH, Chang TC, Chou HH, Hsueh S, Chen CK, Lee SP and Hong JH: Long-term outcome and prognostic factors for adenocarcinoma/adenosquamous carcinoma of cervix after definitive radiotherapy. Int J Radiat Oncol Biol Phys 80(2): 429-436, 2011. PMID: 20542643. DOI: 10.1016/j.ijrobp.2010.02.009

$13 \mathrm{Hu} \mathrm{K}$, Wang W, Liu X, Meng Q and Zhang F: Comparison of treatment outcomes between squamous cell carcinoma and adenocarcinoma of cervix after definitive radiotherapy or concurrent chemoradiotherapy. Radiat Oncol 13(1): 249, 2018. PMID: 30558636. DOI: 10.1186/s13014-018-1197-5

14 Niibe Y, Kenjo M, Onishi H, Ogawa Y, Kazumoto T, Ogino I, Tsujino K, Harima Y, Takahashi T, Anbai A, Tsuchida E, Toita T, Takemoto M, Yamashita $\mathrm{H}$ and Hayakawa K: High-dose-rate intracavitary brachytherapy combined with external beam radiotherapy for stage iiib adenocarcinoma of the uterine cervix in japan: A multi-institutional study of japanese society of therapeutic radiology and oncology 2006-2007 (study of jastro 2006-2007). Jpn J Clin Oncol 40(8): 795-799, 2010. PMID: 20444747. DOI: $10.1093 / \mathrm{jjco} / \mathrm{hyq} 053$

15 Kilic S, Cracchiolo B, Gabel M, Haffty B and Mahmoud O: The relevance of molecular biomarkers in cervical cancer patients treated with radiotherapy. Ann Transl Med 3(18): 261, 2015. PMID: 26605307. DOI: 10.3978/j.issn.2305-5839.2015.10.18

16 Rose PG, Java J, Whitney CW, Stehman FB, Lanciano R, Thomas GM and DiSilvestro PA: Nomograms predicting progression-free survival, overall survival, and pelvic recurrence in locally advanced cervical cancer developed from an analysis of identifiable prognostic factors in patients from nrg oncology/gynecologic oncology group randomized trials of chemoradiotherapy. J Clin Oncol 33(19): 21362142, 2015. PMID: 25732170. DOI: 10.1200/JCO.2014.57.7122

17 Paola D: History of and rational for staging gynaecological cancers. CME J Gynecol Oncol 6: 230-231, 2001.

18 Choudhury KR, Yagle KJ, Swanson PE, Krohn KA and Rajendran JG: A robust automated measure of average antibody staining in immunohistochemistry images. J Histochem Cytochem 58(2): 95-107, 2010. PMID: 19687472. DOI: 10.1369/jhc.2009.953554

19 Roach C, Zhang N, Corigliano E, Jansson M, Toland G, Ponto G, Dolled-Filhart M, Emancipator K, Stanforth D and Kulangara K: Development of a companion diagnostic pd-11 immunohistochemistry assay for pembrolizumab therapy in non-small-cell lung cancer. Appl Immunohistochem Mol Morphol 24(6): 392, 2016. PMID: 27333219. DOI: 10.1097/PAI.0000000000000408

20 Chen SW, Shen WC, Hsieh TC, Liang JA, Hung YC, Yeh LS, Chang WC, Lin WC, Yen KY and Kao CH: Textural features of cervical cancers on FDG-PET/CT associate with survival and local relapse in patients treated with definitive chemoradiotherapy. Sci Rep 8(1): 11859, 2018. PMID: 30089896. DOI: 10.1038/s41598-018-30336-6

21 Chen SW, Liang JA, Hung YC, Yeh LS, Chang WC, Lin WC and Chien CR: Does initial 45gy of pelvic intensity-modulated radiotherapy reduce late complications in patients with locally advanced cervical cancer? A cohort control study using definitive chemoradiotherapy with high-dose rate brachytherapy. Radiol Oncol 47(2): 176-184, 2013. PMID: 23801915. DOI: 10.2478/raon-2013-0011

22 Potter R, Haie-Meder C, Van Limbergen E, Barillot I, De Brabandere M, Dimopoulos J, Dumas I, Erickson B, Lang S, Nulens A, Petrow P, Rownd J, Kirisits C and Group GEW: Recommendations from gynaecological (gyn) gec estro working group (ii): Concepts and terms in $3 \mathrm{~d}$ image-based treatment planning in cervix cancer brachytherapy-3d dose volume parameters and aspects of $3 \mathrm{~d}$ image-based anatomy, radiation physics, radiobiology. Radiother Oncol 78(1): 67-77, 2006. PMID: 16403584. DOI: 10.1016/j.radonc.2005.11.014

23 Grogan M, Thomas GM, Melamed I, Wong FL, Pearcey RG, Joseph PK, Portelance L, Crook $\mathrm{J}$ and Jones KD: The importance of hemoglobin levels during radiotherapy for carcinoma of the cervix. Cancer 86(8): 1528-1536, 1999. PMID: 10526282.

24 Lee KM, Giltnane JM, Balko JM, Schwarz LJ, Guerrero-Zotano AL, Hutchinson KE, Nixon MJ, Estrada MV, Sanchez V, Sanders ME, Lee T, Gomez H, Lluch A, Perez-Fidalgo JA, Wolf MM, Andrejeva G, Rathmell JC, Fesik SW and Arteaga CL: Myc and mcll cooperatively promote chemotherapy-resistant 
breast cancer stem cells via regulation of mitochondrial oxidative phosphorylation. Cell Metab 26(4): 633-647 e637, 2017. PMID: 28978427. DOI: 10.1016/j.cmet.2017.09.009

25 Thomas LW, Lam C and Edwards SW: Mcl-1; the molecular regulation of protein function. FEBS Lett 584(14): 2981-2989, 2010. PMID: 20540941. DOI: 10.1016/j.febslet.2010.05.061

26 Opferman JT, Letai A, Beard C, Sorcinelli MD, Ong CC and Korsmeyer SJ: Development and maintenance of $\mathrm{b}$ and $\mathrm{t}$ lymphocytes requires antiapoptotic mcl-1. Nature 426(6967): 671, 2003. PMID: 14668867. DOI: 10.1038/nature02067

27 Chen L, Willis SN, Wei A, Smith BJ, Fletcher JI, Hinds MG, Colman PM, Day CL, Adams JM and Huang DC: Differential targeting of prosurvival bcl-2 proteins by their bh3-only ligands allows complementary apoptotic function. Mol Cell 17(3): 393403, 2005. PMID: 15694340. DOI: 10.1016/j.molcel.2004. 12.030

28 Harrison L and Blackwell K: Hypoxia and anemia: Factors in decreased sensitivity to radiation therapy and chemotherapy? Oncologist Suppl 5: 31-40, 2004. PMID: 15591420. DOI: 10.1634/theoncologist.9-90005-31

29 Dang CV, Le A and Gao P: Myc-induced cancer cell energy metabolism and therapeutic opportunities. Clin Cancer Res 15(21): 6479-6483, 2009. PMID: 19861459. DOI: 10.1158/ 1078-0432.CCR-09-0889

30 Miller DM, Thomas SD, Islam A, Muench D and Sedoris K: Cmyc and cancer metabolism. Clin Cancer Res 18(20): 55465553, 2012. PMID: 23071356. DOI: 10.1158/1078-0432.CCR12-0977

31 Levens D: You don't muck with myc. Genes Cancer 1(6): 547554, 2010. PMID: 20882108. DOI: 10.1177/1947601910377492

32 Cui F, Fan R, Chen Q, He Y, Song M, Shang Z, Zhang S, Zhu $\mathrm{W}$, Cao J, Guan $\mathrm{H}$ and Zhou PK: The involvement of c-myc in the DNA double-strand break repair via regulating radiationinduced phosphorylation of atm and DNA-pkcs activity. Mol Cell Biochem 406(1-2): 43-51, 2015. PMID: 26049366. DOI: 10.1007/s11010-015-2422-2

33 Wang WJ, Wu SP, Liu JB, Shi YS, Huang X, Zhang QB and Yao $\mathrm{KT}$ : Myc regulation of chk1 and chk2 promotes radioresistance in a stem cell-like population of nasopharyngeal carcinoma cells. Cancer Res 73(3): 1219-1231, 2013. PMID: 23269272. DOI: 10.1158/0008-5472.CAN-12-1408

34 Kim BY, Kwak SY, Yang JS and Han YH: Phosphorylation and stabilization of c-myc by nemo renders cells resistant to ionizing radiation through up-regulation of gamma-gcs. Oncol Rep 26(6): 1587-1593, 2011. PMID: 21874259. DOI: 10.3892/or.2011.1432
35 Papanikolaou V, Iliopoulos D, Dimou I, Dubos S, Kappas C, Kitsiou-Tzeli $\mathrm{S}$ and Tsezou A: Survivin regulation by her2 through nf-kappab and c-myc in irradiated breast cancer cells. J Cell Mol Med 15(7): 1542-1550, 2011. PMID: 20716114. DOI: 10.1111/j.1582-4934.2010.01149.x

36 Jung J, Kim EJ, Chung HK, Park HJ, Jeong SY and Choi EK: $\mathrm{C}$-myc down-regulation is involved in proteasome inhibitormediated enhancement of radiotherapeutic efficacy in non-small cell lung cancer. Int J Oncol 40(2): 385-390, 2012. PMID: 21947247. DOI: 10.3892/ijo.2011.1205

37 Meijer TW, Kaanders JH, Span PN and Bussink J: Targeting hypoxia, hif-1, and tumor glucose metabolism to improve radiotherapy efficacy. Clin Cancer Res 18(20): 5585-5594, 2012. PMID: 23071360. DOI: 10.1158/1078-0432.CCR-12-0858

38 Reynolds JE, Yang T, Qian L, Jenkinson JD, Zhou P, Eastman A and Craig RW: Mcl-1, a member of the bcl-2 family, delays apoptosis induced by c-myc overexpression in chinese hamster ovary cells. Cancer Res 54(24): 6348-6352, 1994. PMID: 7987827.

39 Lee MY, Chou CY, Tang MJ and Shen MR: Epithelialmesenchymal transition in cervical cancer: Correlation with tumor progression, epidermal growth factor receptor overexpression, and snail up-regulation. Clin Cancer Res 14(15): 4743-4750, 2008. PMID: 18676743. DOI: 10.1158/10780432.CCR-08-0234

40 Imai T, Horiuchi A, Wang C, Oka K, Ohira S, Nikaido T and Konishi I: Hypoxia attenuates the expression of e-cadherin via up-regulation of snail in ovarian carcinoma cells. Am J Pathol 163(4): 1437-1447, 2003. DOI: 10.1016/s0002-9440(10)63501-8 41 Yang MH, Wu MZ, Chiou SH, Chen PM, Chang SY, Liu CJ, Teng SC and Wu KJ: Direct regulation of twist by hif-1 alpha promotes metastasis. Nat Cell Biol 10(3): 295-305, 2008. PMID: 18297062. DOI: $10.1038 /$ ncb1691
Received May 2, 2019

Revised May 16, 2019

Accepted May 22, 2019 